\title{
Stress myocardial perfusion imaging vs. stress echocardiography for risk stratification of kidney transplant candidates: Does it even matter?
}

\author{
Aviral Vij, MD, ${ }^{\mathrm{a}, \mathrm{b}}$ and Rami Doukky, MD, MSc, MBA, FASNC ${ }^{\mathrm{a}, \mathrm{b}}$ \\ a Division of Cardiology, Cook County Health, Chicago, IL \\ b Division of Cardiology, Rush University, Chicago, IL
}

Received Nov 18, 2021; accepted Nov 18, 2021

doi: $10.1007 / \mathrm{s} 12350-021-02883-5$

\section{See related article, pp. 2988-2999}

Cardiovascular disease (CVD), rather than endstage kidney disease (ESRD), is the leading cause of death in patients with chronic kidney disease (CKD), accounting for nearly $50 \%$ of all deaths. ${ }^{1,2}$ In the USA, there are over 100,000 patients awaiting kidney transplant and close to 3000 patients are added to the waiting list every month. ${ }^{3}$ With such a high prevalence of CVD in CKD patients and limited number of donor kidneys, several professional societies provide recommendations for cardiac assessment of patients undergoing kidney transplantation in an effort to optimize peri-transplant outcomes. While management of patients with symptomatic coronary artery disease (CAD) is generally well defined, the management and outcome implications of pre-operative cardiac testing in asymptomatic ESRD patients are unclear. The 2014 ACC/AHA peri-operative cardiovascular evaluation guidelines for non-cardiac surgery are not intended for patients undergoing solid organ transplant evaluation. ${ }^{4}$ ESRD patients have a significantly higher prevalence of CAD (up to 53\%) in comparison to non-ESRD patients. ${ }^{5}$ Additionally, cardiac risk assessment of kidney transplant candidates extends beyond the peri-operative period and demands long-term cardiac risk stratification to ensure proper organ allocation to a host with a favorable long-term prognosis.

Reprint requests: Rami Doukky, MD, MSc, MBA, FASNC, Division of Cardiology, Cook County Health, 1901 W. Harrison Street, Chicago, IL 60612; rami_doukky@rush.edu

J Nucl Cardiol 2022;29:3000-2.

$1071-3581 / \$ 34.00$

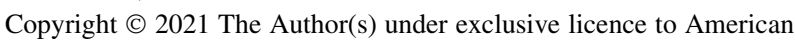
Society of Nuclear Cardiology
It is accepted that CAD surveillance using noninvasive stress testing may be considered in asymptomatic kidney transplantation candidates with no active cardiac conditions based on the presence of multiple CAD risk factors regardless of functional status. ${ }^{6,7}$ The modality of stress testing with either stress echocardiography or stress radionuclide myocardial perfusion imaging (MPI) is left at the discretion of the provider and the expertise of the transplant center. While the sensitivity and specificity of noninvasive stress testing is lower in patients with ESRD compared to the general population, there had been no head-to-head comparisons between different testing modalities in this population. However, the published data suggest a comparable diagnostic accuracy between SPECT MPI and dobutamine stress echocardiography (DSE). The literature provides even less insight into the comparative prognostic utility of these tests. ${ }^{8,9}$

In this issue of the journal, Bautz et al directly compared, in the same patients, the prognostic utility of SPECT MPI and DSE in 229 kidney transplant candidates who underwent both tests within 1 month. ${ }^{10}$ The primary endpoint was a composite of nonfatal myocardial infarction (MI) or all-cause death and the secondary endpoint was a composite of nonfatal and fatal MI or coronary revascularization (not triggered by baseline MPI or DSE). The threshold for an abnormal MPI scan (SSS $\geq 1$ ) was lower than the traditional cut-off (SSS $\geq$ 4) commonly used in the literature. Abnormal MPI or abnormal DSE were observed in $15.7 \%$ and $16.2 \%$ of subjects, respectively. MPI and DSE showed good overall agreement in $88.6 \%$ of cases at rest (rest perfusion/rest regional wall motion abnormalities [RWMA]) and $80 \%$ at stress (stress perfusion/stress RWMA). Contradictory results were noted in $9 \%$ of resting images and $14 \%$ of stress images. The study was not designed or powered to evaluate the diagnostic accuracy of either modality. 
The primary (nonfatal MI or all-cause death) and secondary (fatal/nonfatal MI or coronary revascularization) endpoints were observed in $70(30.6 \%)$ and 24 $(10.5 \%)$ patients. Abnormal MPI was associated with a statistically significant increase in the primary endpoint (adjusted hazard ration [HR] 1.77; 95\% confidence interval $[\mathrm{CI}] 1.02-3.08 ; P=.043$ ), whereas abnormal DSE was not associated with a statistically significant increase (adjusted HR 1.36; 95\% CI 0.78-2.37; $P=\mathrm{NS}$ ). On the other hand, both abnormal MPI (adjusted HR $3.21 ; 95 \%$ CI 1.35-7.61; $P=.008)$ and abnormal DSE (adjusted HR 2.67; 95\%CI 1.15-6.20; $P=.022$ ) were associated with a statistically significant increase in the secondary endpoint. Combining the two imaging modalities only modestly improved the prognostic value for the primary (adjusted HR 1.92; 95\% CI 1.17-3.17; $P=.01$ ) and secondary (adjusted HR 3.45; 95\%CI 1.49$8.01 ; P=.04)$ endpoints. It is interesting to note that the effect size (HR) of abnormal stress MPI seems to be numerically greater than DSE. A greater HR may suggest that stress MPI is more effective than DSE in risk stratification. It is unclear whether this observation is statistically significant, as interaction analysis between the stress modality and the study finding (normal vs. abnormal) was not performed. However, noting a substantial overlap between confidence intervals suggests that there is no significant interaction.

Patients who underwent kidney transplant during the follow-up had lower incidence of the primary $(21.9 \%$ vs. $54.2 \%)$ and secondary $(8.9 \%$ vs. $13.3 \%)$ endpoints. However, these differences were not statistically significant when kidney transplant was used as a time-dependent exposure.

We commend the authors for performing this first of its kind study, wherein all patients underwent both MPI and DSE within 1 month. The results of this study confirmed an existing consensus that both abnormal stress MPI and DSE can predict adverse cardiac events in ESRD patients undergoing kidney transplant evaluation. The study informs us that there is no additive value for performing both stress testing modalities in these patients. Although it was not demonstrated statistically, the study suggests that SPECT MPI appears to outperform DSE in prognostication. This observation can be explained by the ischemic cascade physiology. It is established that myocardial perfusion abnormality occurs at lower threshold of ischemia than RWMA (more sensitive). Thus, MPI may have better identified patients at risk. Larger studies are needed to further investigate this observation.

While Inflammation and oxidative stress with CKD have been linked to the pathogenesis of plaque formation and plaque rupture leading to acute $\mathrm{MI},{ }^{11}$ heart failure remains the most common manifestation of CVD in this population. ${ }^{12}$ Besides the increased prevalence of traditional risk factors, ${ }^{13}$ non-traditional risk factors, such as vascular calcifications, ${ }^{14,15}$ circulating proinflammatory mediators, ${ }^{16,17}$ and pathological myocardial fibrosis leading to hypertrophy (uremic cardiomyopathy $)^{18}$ contribute significantly to cardiovascular risk and mortality in these patients. CAD remains one part of this puzzle and we may be too focused on identifying CAD. Although there are certain scenarios where identifying severe and extensive CAD in asymptomatic ESRD patients may have important implications on outcome and management, there is no proven benefit of routine revascularization in CKD patients. ${ }^{19}$ Importantly, identifying ESRD patients with unacceptably high CVD risk and limited life expectancy would be crucial in determining eligibility for kidney transplantation and better allocate scarce donor organs.

Reflex coronary revascularization in asymptomatic patients with abnormal stress test is not the standard of care in general population and we believe this should not be the case in kidney transplant candidates either. Optimizing medical management of $\mathrm{CAD}$ and heart failure remains the mainstay of management in these patients. Undoubtedly, pre-operative stress testing has shown prognostic value in ESRD patients undergoing transplant evaluation. However, it is still unclear how to use this prognostic information in decision-making. While we improve the management of traditional risk factors and aim for early kidney transplant, additional research is needed to find ways to incorporate this prognostic information into patient management to achieve the eventual goal of improving outcomes. Realizing this goal requires large-scale comparative outcome studies and randomized clinical trials addressing the value of medical therapy and coronary revascularization in patients undergoing kidney transplantation.

\section{Disclosure}

None.

\section{References}

1. Webster AC, Nagler EV, Morton RL, Masson P. Chronic kidney disease. Lancet 2017;389:1238-52.

2. Thompson S, James M, Wiebe N, Hemmelgarn B, Manns B, Klarenbach S. Cause of death in patients with reduced kidney function. J Am Soc Nephrol 2015;26:2504-11.

3. Alkoutami GS, Reeves WC, Movahed A. The frequency of atrioventricular block during adenosine stress testing in young, middle-aged, young-old, and old-old adults. Am J Geriatr Cardiol 2001;10:159-61. 
4. Fleisher LA, Fleischmann KE, Auerbach AD, Barnason SA, Beckman JA, Bozkurt B, et al. 2014 ACC/AHA guideline on perioperative cardiovascular evaluation and management of patients undergoing noncardiac surgery: Executive summary: A report of the American College of Cardiology/American Heart Association Task Force on Practice Guidelines. Circulation 2014;130:2215-45.

5. Ohtake T, Kobayashi S, Moriya H, Negishi K, Okamoto K, Maesato K, et al. High prevalence of occult coronary artery stenosis in patients with chronic kidney disease at the initiation of renal replacement therapy: An angiographic examination. J Am Soc Nephrol 2005;16:1141-8.

6. Lentine KL, Costa SP, Weir MR, Robb JF, Fleisher LA, Kasiske $\mathrm{BL}$, et al. Cardiac disease evaluation and management among kidney and liver transplantation candidates. J Am Coll Cardiol 2012;60:434-80.

7. Doukky R, Fughhi I, Campagnoli T, Wassouf M, Kharouta M, Vij A, et al. Validation of a clinical pathway to assess asymptomatic renal transplant candidates using myocardial perfusion imaging. J Nucl Cardiol 2018;25:2058-68.

8. Kassab K, Doukky R. Cardiac imaging for the assessment of patients being evaluated for kidney transplantation. J Nucl Cardiol 2021. https://doi.org/10.1007/s12350-021-02561-6.

9. Parikh K, Appis A, Doukky R. Cardiac imaging for the assessment of patients being evaluated for kidney or liver transplantation. $\mathrm{J}$ Nucl Cardiol 2015;22:282-96.

10. Bautz J, Stypmann J, Reiermann S, Pavenstädt HJ, Suwelack B, Stegger L, et al. Prognostic implication of myocardial perfusion and contractile reserve in end-stage renal disease: A direct comparison of myocardial perfusion scintigraphy and dobutamine stress echocardiography. J Nucl Cardiol 2021. https://doi.org/10. 1007/s12350-021-02844-y.

11. Bhatt DL. Anti-inflammatory agents and antioxidants as a possible "third great wave" in cardiovascular secondary prevention. Am J Cardiol 2008;101:4d-13d.
12. Hurwitz GA, Powe JE, Driedger AA, Finnie KJ, Laurin NR, MacDonald AC. Dipyridamole combined with symptom-limited exercise for myocardial perfusion scintigraphy: Image characteristics and clinical role. Eur J Nucl Med 1990;17:61-8.

13. Major RW, Cheng MRI, Grant RA, Shantikumar S, Xu G, Oozeerally I, et al. Cardiovascular disease risk factors in chronic kidney disease: A systematic review and meta-analysis. PLoS ONE 2018;13:e192895.

14. Goodman WG, Goldin J, Kuizon BD, Yoon C, Gales B, Sider D, et al. Coronary-artery calcification in young adults with end-stage renal disease who are undergoing dialysis. $\mathrm{N}$ Engl $\mathrm{J}$ Med 2000;342:1478-83.

15. Ellis CL, O'Neill WC. Questionable specificity of histologic findings in calcific uremic arteriolopathy. Kidney Int 2018;94:3905 .

16. Amdur RL, Feldman HI, Dominic EA, Anderson AH, Beddhu S, Rahman M, et al. Use of measures of inflammation and kidney function for prediction of atherosclerotic vascular disease events and death in patients with CKD: Findings from the CRIC Study. Am J Kidney Dis 2019;73:344-53

17. Zoccali C, Vanholder R, Massy ZA, Ortiz A, Sarafidis P, Dekker FW, et al. The systemic nature of CKD. Nat Rev Nephrol 2017;13:344-58.

18. Alhaj E, Alhaj N, Rahman I, Niazi TO, Berkowitz R, Klapholz M. Uremic cardiomyopathy: An underdiagnosed disease. Congest Heart Fail 2013;19:E40-5.

19. Bangalore S, Maron DJ, O’Brien SM, Fleg JL, Kretov EI, Briguori $\mathrm{C}$, et al. Management of coronary disease in patients with advanced kidney disease. N Engl J Med 2020;382:1608-18.

Publisher's Note Springer Nature remains neutral with regard to jurisdictional claims in published maps and institutional affiliations. 